\title{
Intoxicación por alcoholes
}

\section{Alcohol intoxication}

\section{J. Roldán ${ }^{1}$, C. Frauca ${ }^{2}$, A. Dueñas ${ }^{3}$}

\section{RESUMEN}

La intoxicación etílica es la primera toxicomanía en muchos países del mundo. Afecta a todos los tramos de edad, en los dos sexos y en casi todos los grupos sociales. La mortalidad asociada sólo a la intoxicación etílica aguda es excepcional, pero puede ser un importante factor si coexiste con ingesta de otras drogas de abuso. Es responsable directo de más de la mitad de los accidentes de tráfico. El diagnóstico es fácil por la anamnesis y la clínica y se puede confirmar determinando el nivel de etanol en sangre. El tratamiento es de sostén, intentando proteger al paciente de complicaciones secundarias.

El metanol o alcohol de quemar se utiliza como disolvente, encontrándose también como adulterante de bebidas alcohólicas. La intoxicación vía oral es la más frecuente. Oxidado en el hígado a través de la enzima alcohol deshidrogenasa, la toxicidad se debe a sus metabolitos, formaldehído y ácido fórmico. La clínica consiste fundamentalmente en cefalea, náuseas, vómitos, hipotensión y depresión del SNC. El nervio óptico es especialmente sensible pudiendo producirse una ceguera total e irreversible.

El etilenglicol se utiliza como disolvente y anticongelante; la toxicidad se debe a la acumulación de sus metabolitos. La clínica incluye síntomas comunes con la intoxicación metílica. Puede ocurrir fallo renal por necrosis tubular y depósito de cristales de oxalato.

Palabras clave. Etanol. Metanol. Intoxicación. Fomepizol. Etilenglicol.

\section{ABSTRACT}

Alcohol intoxication is the principal drug addiction in many countries of the world. It affects all age groups, both sexes and almost all social groups. Mortality associated with acute alcohol poisoning on its own is exceptional, but it can be an important factor if it coexists with recreational drugs. It is directly responsible for more than half of traffic accidents. Diagnosis is easy by means of anamnesis and clinical examination, and can be confirmed by determining the level of ethanol in the bloodstream. Supportive care is the best therapy in order to protect the patient from secondary complications.

Methanol, or alcohol fuel, is used as a solvent, and can also be found as an adulterant of alcoholic drinks. Poisoning by oral means is the most frequent. Oxidized in the liver through dehydrogenase enzyme alcohol, toxicity is due to its metabolites, formaldehyde and formic acid. The clinical picture basically consists of cephalea, nausea, vomiting, hypotension and depression of the central nervous system. The optic nerve is especially sensitive, with total and irreversible blindness as a possible result.

Ethylenglicol is used as a solvent and as an antifreeze; toxicity is due to an accumulation of its metabolites. The clinical picture includes symptoms that are held in common with methylalcohol intoxication. Kidney failure due to tubular necrosis and the deposit of oxalate crystals can occur.

Key words. Ethanol. Methanol. Poisoning. Fomepizol. Ethylenglicol.

ANALES Sis San Navarra 2003; 26 (Supl. 1): 129-139.

1. Servicio de Medicina Intensiva. Hospital de Navarra.

2. Servicio de Urgencias. Hospital de Navarra.

3. Unidad Regional de Toxicología Clínica. Hospital del Río Ortega. Valladolid.

\section{Correspondencia:}

José Roldán Ramírez

Servicio de Medicina Intensiva

Hospital de Navarra

Irunlarrea, 3

31008 Pamplona

Tfno. 948422100 Ext: 2141

Fax 948422303

E-mail: joseroldanetna@hotmail.com 


\section{ETANOL}

El etanol, de fórmula química $\mathrm{CH}_{3} \mathrm{CH}_{2} \mathrm{OH}$, es el principal componente de las bebidas alcohólicas; éstas se obtienen por fermentación o destilación. Según se trate de un procedimiento u otro, se conseguirán bebidas de diferente graduación; así por ejemplo, vinos, cervezas o champán surgen a partir de la fermentación de frutas o granos, mientras que habrá que recurrir a la destilación para lograr ginebra, whisky, ron, etc.

El alcohol es una sustancia que actúa como depresora del SNC. Sus efectos son una consecuencia directa de su acción sobre las membranas celulares y sobre los neurotransmisores. Se potencian cuando el etanol se consume junto a otras drogas: sedantes, hipnóticos, anticonvulsionantes, antidepresivos, tranquilizantes, analgésicos, opiáceos, etc.

El alcohol produce tolerancia. Afecta el SNC, y produce además hipoglucemias, hepatitis aguda, trastornos cardíacos, rabdomiolisis, etc.

El alcohol es probablemente la droga más antigua que se conoce ${ }^{1}$. Así:

- Una tablilla cuneiforme del 2200 a.C. recomienda la cerveza como tónico para las mujeres en estado de lactancia. El código del rey babilónico Hamurabi amparaba a los bebedores de cerveza y vino de palma, y hacía ejecutar a la tabernera que rebajara la calidad de la bebida.

- Los griegos rendían culto a Dionisio y ofrecían bebidas alcohólicas a sus dioses y a los soldados antes de que estos entraran en combate. También ellos las utilizaban para facilitar sus relaciones: constituían el eje de los denominados symposia (banquetes celebrados con fines recreativos en los que intercambiaban ideas filosóficas, políticas, etc., mientras ingerían vino, cerveza e hidromiel).

- En la Biblia, el vino aparece en los episodios de Noé.

- Los romanos apreciaron enormemente el vino y contribuyeron a la difusión de la vid por toda Europa, así como a afianzar la reglamentación de la viticultura.

- Parece ser que fueron los árabes quienes descubrieron la destilación y de cuya mano se introdujo este proceso en el continente europeo.

- El consumo de bebidas alcohólicas se asoció durante la Edad Media con salud y bienestar. De hecho, el alcohol adoptó ese nombre a finales del siglo XVI pues hasta entonces se lo conocía con el elocuente apelativo de aqua vitae.

- Será a partir de la Revolución Industrial del siglo XIX cuando el consumo de esta sustancia alcance niveles hasta entonces desconocidos y que se verán notablemente incrementados desde la segunda mitad de esta centuria.

\section{Patrones de consumo}

El alcoholismo es la primera toxicomanía en muchos países del mundo. Afecta a un gran número de individuos, en general adultos, pero también y cada vez más los adolescentes van viéndose afectados, no sólo por procesos de alcoholización sino también por problemas relacionados con los consumos de alcohol, aunque no medie la dependencia (accidentes, problemas familiares, escolares, intoxicaciones graves...). Es la droga más consumida en todos los tramos de edad, en los dos sexos y en casi todos los grupos sociales.

Con respecto a los más jóvenes, esta sustancia se consume muy por delante de otras drogas ilegales.

\section{Consumo en España}

Según datos del Observatorio Español sobre Drogas (1999), el alcohol -desde su "privilegiada" posición de sustancia psicotrópica no sólo legal, sino además socializada- ocupa un primer rango en los consumos de los españoles mayores de 14 años: un $49 \%$ reconoce su uso en el último mes, mientras que un $15 \%$ admite su consumo diario.

Se ha producido un cambio significativo en los patrones de consumo en los últimos diez-quince años. Del consumo ocasional, especialmente asociado a las comidas en familia y pequeñas reuniones, se ha pasado a un consumo que, protagonizado por jóvenes, centra exclusivamente su actividad en los fines de semana y otros períodos de ocio. Esta ruptura con la tra- 
dicional forma de consumo se manifiesta también en la elección de las bebidas. Frente al vino, surge con fuerza la cerveza, los combinados y los licores de alta graduación. (Alarma por la quinta del botellón: La Vanguardia, 10/2/02). Centenares de jóvenes se reúnen en parques y plazas las noches de jueves, viernes y sábado para montarse su propia fiesta callejera, con el alcohol como principal protagonista. Unas veladas etílicas conocidas como "el botellón”. Los servicios de Urgencia de Madrid atienden cada fin de semana a 60 chicos con intoxicación etílica aguda.

Se aprecia una creciente precocidad en el inicio de su consumo: a los 11 años, un $3,5 \%$ ya ha probado bebidas alcohólicas.

En los últimos años se aprecia un incremento en el consumo de alcohol entre las mujeres, observándose por primera vez un mayor consumo entre las mujeres jóvenes (15 a 18 años) que los registrados entre varones de su misma edad.

Frente a la tendencia decreciente en los consumos globales de alcohol durante los últimos años, en 1997 se aprecia un incremento del $1,5 \%$ respecto al año anterior, aumentando los consumos de sidra, vino y cerveza frente a la disminución del consumo de licores.

\section{Aspectos socioeconómicos}

El alcohol ha pasado a ser, junto con el tabaco, una de las principales causas individuales de enfermedad prevenible en
España. Es responsable directo de más de la mitad de los accidentes de tráfico, que constituyen la primera causa de muerte en los menores de 30 años (El País 26/12/00).

En un estudio sobre el coste económico del alcohol para la sociedad, se puede leer que la baja productividad derivada del alcohol se cuantifica en 1.730 millones de euros, 288.069 millones de pesetas. Pero sin duda lo más impresionante es el número de años potenciales de vida que se pierden en España por problemas de alcohol: 224.000 (La Vanguardia 10/02/02).

Según datos de la DGT (Dirección General de Tráfico) se estima que en el 38\% de los siniestros están presentes el alcohol o las drogas. En mayo de 1999 se disminuyó la tasa de alcoholemia permitida en sangre: para conductores de vehículos deberá estar por debajo de $0,5 \mathrm{~g} / \mathrm{L}(0,25 \mathrm{mg} / \mathrm{L}$ en aire espirado) y para conductores noveles o profesionales por debajo de $0,3 \mathrm{~g} / \mathrm{L}$.

El Instituto Nacional de Estadística informa de que el consumo de alcohol en España es responsable del $46 \%$ de las muertes por homicidio y del $25 \%$ de las debidas a suicidio. También es responsable del $40 \%$ de los fallecimientos por accidentes de tráfico.

Muchos productos domésticos contienen etanol y esto hace posible que sea el responsable de algunas intoxicaciones accidentales en la infancia ${ }^{2}$ (Tabla 1).

Tabla 1. Porcentaje de contenido en etanol de distintos productos domésticos y bebidas.

\begin{tabular}{|c|}
\hline Productos domésticos \\
\hline 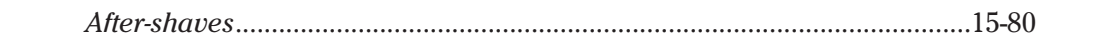 \\
\hline Fármacos antialérgicos y catarrales ........................................................5-16 \\
\hline 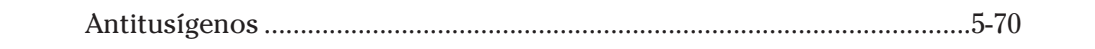 \\
\hline 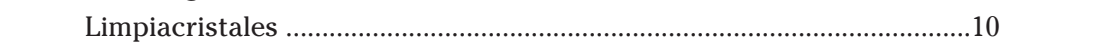 \\
\hline 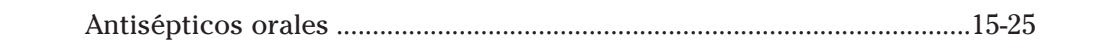 \\
\hline 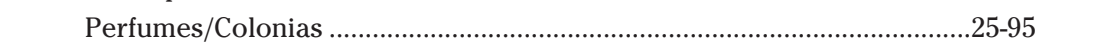 \\
\hline Bebidas \\
\hline 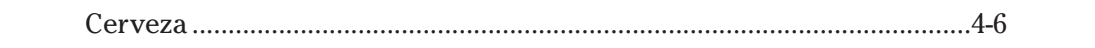 \\
\hline 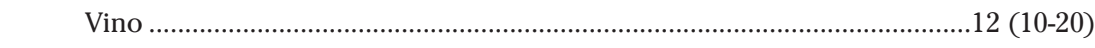 \\
\hline Licores \\
\hline
\end{tabular}




\section{Fisiopatología ${ }^{3}$}

La cantidad de alcohol ingerida con una bebida determinada es proporcional a su grado alcohólico y al volumen ingerido, multiplicado por 0,8 que es la densidad del alcohol:

$$
\mathrm{Gr}=\text { graduación } \mathrm{x} \text { Volumen } \mathrm{x} \text { 0,8 / } 100
$$

Estimación de niveles de etanolemia en $\mathrm{g} / \mathrm{l}:$

- Ingesta en $\mathrm{ml} x$ (graduación de la bebi$\mathrm{da} / 100) \times 0,8 \mathrm{~g} / \mathrm{ml}$

- Volumen de distribución $(0,6$ L/Kg) x Peso en Kg.

Tras su ingestión es rápidamente absorbido por la mucosa del estómago en un $30 \%$ y después por el intestino delgado proximal en el $70 \%$ restante. Se distribuye por los tejidos siguiendo el espacio del agua corporal y es casi completamente oxidado en el hígado siguiendo una cinética de orden cero (independiente de la concentración) a un ritmo de 15 a $20 \mathrm{mg} / \mathrm{dl} /$ hora dependiendo del peso corporal.

Los alcohólicos crónicos pueden metabolizar el alcohol con doble rapidez. Sufre un primer paso débil metabólico en la mucosa gástrica que contiene alcoholato deshidrogenasa y después difunde a todo el organismo por su coeficiente grasa/agua favorable. Su degradación es esencialmente por oxidación hepática en un $90 \%$ y un $10 \%$ puede ser eliminado por vías accesorias como son el riñón y el pulmón.

El nivel de alcoholemia es el resultado de la absorción digestiva, de la distribución tisular, de la oxidación y de su eliminación. Se determina, bien por la medida directa de la concentración de etanol en sangre o bien indirectamente por la medida de la tasa en el aire espirado ${ }^{4,5}$.

\section{Metabolismo del etanol}

El etanol se convierte en acetaldehído por la acción de tres enzimas:

- Alcohol deshidrogenasa (ADH). En no alcohólicos el 90-95\% de la oxidación del etanol se realiza por medio del ADH. Las mujeres tienen un nivel más bajo de actividad $\mathrm{ADH}$ gástrico que el hombre y esto contribuye a que las mujeres tengan valores más altos de alcoholemia que los hombres.

- Sistema oxidativo microsomal del etanol (MEOS), es un sistema enzimático dependiente del citocromo P-450 y contribuye con el 5-10\% a la oxidación del etanol en bebedores moderados, pero su actividad aumenta significativamente en bebedores crónicos hasta un 25\%. Cuando los niveles de etanolemia son altos se activa el sistema MEOS que contribuye a disminuir rápidamente los niveles.

- Sistema catalasa-peroxidasa dependiente del peróxido de oxígeno, la contribución a la oxidación del etanol es mínimo.

Etanol $\rightarrow$ Acetaldehído $\rightarrow$ Acetato $\rightarrow$ Acetilcoenzima $\mathrm{A} \rightarrow \mathrm{CO}_{2}+\mathrm{H}_{2} \mathrm{O}$

\section{Clínica de la intoxicación etílica aguda}

Las manifestaciones más importantes de la intoxicación etílica aguda $^{2}$ son los cambios conductuales desadaptativos como la desinhibición de impulsos sexuales o agresividad, labilidad emocional, deterioro de la capacidad de juicio y de la actividad social o laboral, lenguaje farfullante, descoordinación, marcha inestable, rubor facial, cambio del estado de ánimo, irritabilidad, locuacidad y disminución de la capacidad de atención. La conducta habitual del sujeto puede acentuarse o alterase. A veces se asocia una amnesia de los acontecimientos durante la intoxicación.

Factores como la existencia de tolerancia, el tipo y la cantidad de bebida ingerida, la rapidez del consumo, toma simultánea o no de alimentos, circunstancias ambientales, personalidad, consumo de algún medicamento..., influirán de forma notable en las características de la embriaguez.

Los casos más graves de intoxicación determinan pérdida de conciencia, coma e incluso, muerte por depresión cardiorrespiratoria.

Se considera dosis tóxica en adultos a 5 $\mathrm{g} / \mathrm{Kg}$ y $3 \mathrm{~g} / \mathrm{Kg}$ en niños.

Aunque los niveles en sangre en no bebedores se correlacionan con los síntomas en bebedores crónicos se requieren niveles más altos para alcanzar estados similares de intoxicación. 
La intoxicación puede manifestarse de una forma u otra según los niveles sanguíneos de etanol:

- 20-30 mg/dl: se afecta el control fino, el tiempo de reacción y hay deterioro de la facultad crítica y del estado de humor.

- 50-100 mg/dl: hay deterioro leve o moderado de las funciones cognitivas, dificultad para grandes habilidades motoras.

- 150-200 mg/dl: el 50\% de las personas pueden estar muy intoxicadas con ataxia y disartria, grave deterioro mental y físico, euforia, combatividad.

- 200-300 mg/dl: náuseas, vómitos, diplopia, alteraciones del estado mental.

- 300 mg/dl: generalmente produce coma, además hipotensión e hipotermia en personas que no beben habitualmente.

- 400-900 mg/dl: rango letal, independientemente de que sea o no un alcohólico crónico.

La depresión vascular se debe a factores vasodepresores centrales y a depresión respiratoria. La hipotermia está favorecida por la primera fase de vasodilatación cutánea y sensación de calor, con aumento de la pérdida calórica y también pérdida del termostato central de la temperatura. La intoxicación alcohólica junto con las drogas de abuso y los trastornos psiquiátricos son las causas más frecuentes de hipotermia.

\section{Diagnóstico}

El diagnóstico se basa en una anamnesis que constate la ingestión de bebida alcohólica, la eliminación de otras causas responsables de síntomas similares: hipoglucemia, traumatismo craneoencefálico, encefalopatía metabólica, infecciones, hipotermia, shock..., y de la evolución: regresión de los síntomas a las 3-6 horas siguientes.

El uso de rutina de medir los niveles de etanolemia en el Servicio de Urgencias es contradictorio, pero se debería realizar en la evaluación de un paciente con el nivel de conciencia disminuido.

Hay que tener en cuenta que antes de sacar sangre se debe limpiar la piel con suero fisiológico.
El etanol también se puede determinar en orina.

\section{Tratamiento}

- El tratamiento de la intoxicación etílica aguda $^{5}$ es de sostén, intentando proteger al paciente de traumatismos secundarios. Vigilancia estrecha de las posibles complicaciones como vómitos y trastornos respiratorios.

- Exploración física adecuada y completa, para evitar dejar pasar una patología acompañante.

- Ante coma con aspiración o convulsiones se hará intubación y ventilación mecánica, así como tratamiento anticonvulsivante.

- Se administrará glucosa intravenosa en todo paciente con alteración del estado mental previa tira reactiva y se trasladará al hospital. Se debe de atender en un lugar tranquilo y vigilarlo. Adoptar posición de seguridad. Control neurológico frecuente.

- Constantes vitales tomadas frecuentemente para despistar otro proceso grave asociado. Vigilar vía aérea, respiración y situación hemodinámica.

- Despitaje de TCE u otro traumatismo. Indagar por la existencia de otros posibles tóxicos. Utilizar naloxona y flumazenil en caso de duda.

- Fluidoterapia. El paciente intoxicado tiene frecuentemente depleción del volumen secundario a que el etanol induce la diuresis y vómitos junto con una pobre ingesta oral. Vigilar la hipoglucemia y acidosis metabólica en los niños. Si hipoglucemia en el niño: $0,5 \mathrm{mg} / \mathrm{Kg}$ de glucosa intravenosa, seguida de perfusión de glucosado al 10\%. En supuestos alcohólicos crónicos se administrará tiamina 100 mg im para evitar el Síndrome de Wernicke- Korsakoff.

- Mantenerlo bien abrigado para evitar la hipotermia.

- Si agitación psicomotriz, clorazepato dipotásico $100 \mathrm{mg}$ oral ó $10 \mathrm{mg} / \mathrm{min}$ intravenoso, hasta que se inicie un principio de sedación y valorar si es necesaria la contención física. Son frecuentes 
en estos enfermos las complicaciones respiratorias derivadas de un exceso de sedación, realizándose una estrecha monitorización cardiorrespiratoria, evitando siempre el exceso de fármacos sedantes por las frecuentes depresiones respiratorias que se producen. También en casos más difíciles se puede pautar butirofenonas. En casos de estados agudos de angustia pautar carbamatos, benzodiacepinas.

- En caso de intoxicaciones letales, etanolemia $>500 \mathrm{mg} / \mathrm{dl}$, se puede considerar la hemodiálisis para aumentar la tasa de eliminación de etanol, especialmente en niños, cuando no mejoran con el tratamiento de soporte y ante convulsiones persistentes, trastornos metabólicos, hipoglucemia persistente y posibilidad de intoxicación con otras drogas.

- Indicaciones de TAC craneal: existencia de focalidad. Crisis convulsiva. Persistencia o agravación del estado mental tras observación de 3-6 horas.

- Dejarlo en sala vigilada por enfermería, con familiares y amigos, durante varias horas.

- Cuando mejore y se pueda valer por sí mismo se le dará el alta médica. Mejor acompañado de familiares o amigos; en caso de menores de edad es obligatorio avisar a sus padres o en su defecto a familiares próximos mayores de edad.

\section{Criterios de ingreso en observación}

- Intoxicación en niños.

- Intoxicación que precise importante tratamiento de soporte.

- Presencia de insuficiencia cardiaca, renal, hepática.

- Ingestión de otros tóxicos o medicamentos que pueden potenciar los efectos del alcohol.

- Exposición prolongada al frío o al calor.

- Los que presenten: HDA, neumonía o encefalopatía.

- Los que presenten alteraciones metabólicas: K menor de $3 \mathrm{mEq} / \mathrm{l}$ o con efectos cardiacos, calcio menor de 1,5 mEq/l ionizado o con efectos cardiacos o neurológicos.
- Complicaciones del alcoholismo: pancreatitis, infección, etc.

\section{No son útiles en la intoxicación etílica aguda}

- El lavado gástrico, por la rápida absorción del etanol.

- Carbón activado, no bloquea a etanol, ni catárticos. Se deberían considerar sólo si hay sospecha de ingesta conjunta de otras drogas.

- La administración de vitamina B6 intavenosa, la cafeína, u otros remedios caseros: "café con sal".

- La diuresis forzada.

- El flumazenilo y la naloxona.

- Antídotos. En la actualidad, no se cuenta con agentes de utilidad clínica que puedan revertir todos los efectos farmacológicos del alcohol. Hay un compuesto conocido como RO15-4513 que antagoniza de manera parcial los efectos del alcohol a nivel del receptor del tipo A del GABA y, por tanto, disminuye los efectos sedantes, ansiolíticos y de transtorno motor del alcohol, pero no los efectos intoxicantes letales. El RO15-4513 está limitado en la actualidad por sus toxicidades, entre ellas los efectos proconvulsivos y ansiógenos. Aunque no se conoce la utilidad de un antagonista del alcohol, su función más probable sería corregir la sobredosis ${ }^{6}$.

\section{METANOL}

El metanol es un líquido incoloro y volátil a temperatura ambiente. Por sí mismo es inofensivo, pero sus metabolitos son tóxicos. Su uso es muy habitual en la industria, laboratorios y en el propio hogar con alcohol de quemar o formando parte de productos comerciales. Además, existe un uso fraudulento de esta sustancia como sustituto del etanol en bebidas alcohólicas fabricadas clandestinamente. La vía más habitual de intoxicación aguda es la oral. La dosis mínima letal se considera que es de $30 \mathrm{ml}$ de metanol puro, aunque en la bibliografía se barajan cifras muy dispares en relación con la dosis tóxica (que produce ceguera) y/o mortal, existiendo una gran variabilidad interindividual a este res- 
pecto. Independientemente de cual sea la dosis letal la mortalidad por metanol es muy alta (hasta un 50\%).

\section{Fisiopatología}

El metanol es biotransformado en el hígado $^{7-9}$, oxidándose merced a la alcoholdeshidrogenasa dando formaldehído, el cual es rápidamente convertido, por la aldehídodeshidrogenasa, en ácido fórmico. Éste último, mediante una oxidación dependiente del folato, se convertirá en anhídrido carbónico y agua. En la toxocinética del metanol interesa fijarse en un detalle: el etanol tiene una afinidad por la alcoholdeshidrogenasa unas $10-20$ veces superior al metanol, de ahí su eficacia como antídoto.

Los efectos tóxicos de la sobredosis de metanol se deben a la formación de estos dos metabolitos, ya que el alcohol metílico per se sólo produce depresión del SNC. Aunque en un principio se señaló que el formaldehído desempeñaba un papel preponderante en la toxicidad ocular, estudios posteriores demostraron que, por su rápida transformación en ácido fórmico, carecía de tal papel patogénico. Por tanto, el ácido fórmico será el principal responsable de la toxicidad ocular, y de la acidosis metabólica anión gap elevado.

\section{Clínica}

El inicio de la sintomatología varía extraordinariamente, entre 40 minutos a 72 horas, aunque lo habitual suele ser que aparezcan los síntomas en las primeras 1224 horas (antes el enfermo padece una especie de borrachera) ${ }^{7-9}$. El criterio clínico de intoxicación grave por metanol es la ceguera. Los síntomas y signos de la intoxicación por vía oral generalmente afectan al SNC, tracto gastrointestinal, ojos y pueden agruparse en:

- Sistema Nervioso Central: en la intoxicación leve o moderada se produce cefalea, mareo, letargia, ataxia o simplemente un estado de embriaguez similar al de la intoxicación etílica. En casos graves pueden aparecer convulsiones, coma y edema cerebral.
- Afectación ocular: hay pérdida de la agudeza visual con edema de papila. Además, puede aparecer nistagmus y alteraciones de los reflejos pupilares. Asimismo, puede desarrollarse pérdida de visión y ceguera irreversible por atrofia del nervio óptico.

- Gastrointestinales: debido a su acción ligeramente irritante, se producen náuseas, vómitos y dolor abdominal; en un segundo tiempo pueden presentarse manifestaciones clinico-biológicas de pancreatitis aguda, con aumento de transaminasas y enzimas pancreáticos.

- Otras manifestaciones: la aparición, a veces poco clara, de disnea o taquipnea, puede reflejar la presencia de una acidosis metabólica normoclorémica con hiato aniónico elevado. En ocasiones se percibe un olor de la orina a formaldehído.

\section{Diagnóstico}

El diagnóstico temprano de la certeza de una intoxicación aguda por un producto que contenga metanol ${ }^{9}$ viene dado por la demostración analítica de su presencia en el plasma del paciente, técnica por otra parte no disponible en la mayoría de los hospitales españoles. Debido al período de latencia y/o a los escasos síntomas iniciales, fáciles de confundir con una simple intoxicación etílica, se deberá pensar en intentos de suicidio o en accidentes domésticos cuando el paciente presente acidosis metabólica intensa y rebelde al tratamiento y también se le aprecie aumento del hiato aniónico y osmolar.

El diagnóstico diferencial debe plantearse con todas aquellas causas que produzcan una acidosis metabólica con anión gap y osmol gap aumentado (intoxicación por salicilatos, etilenglicol, cetoacidosis alcohólica, etc.).

Hoy en día se puede hacer un diagnóstico rápido de la ingesta de metanol pura (sin asociar etanol) mediante kits comerciales basados en la detección de alcohol deshidrogenasa y alcohol oxidasa.

En todos los casos de sospecha de intoxicación o para valorarla, será necesaria solicitar una analítica general, y una gaso- 
metría, y calcular el anión gap (> 16 $\mathrm{mmol} / \mathrm{L}$ ) y el osmolgap (> $25 \mathrm{mOsm} / \mathrm{Kg}$ ). También deberemos solicitar niveles de etanol, para determinar si hubo coingesta.

La dosis tóxica es de 10-30 ml, considerándose potencialmente letal una dosis de 60-240 ml. Los niveles plasmáticos tóxicos son superiores a $0,2 \mathrm{~g} / \mathrm{l}$, y potencialmente mortales los que superan $1 \mathrm{~g} / \mathrm{L}$.

El pronóstico mejora si la dosis ingerida ha sido fraccionada en el tiempo, si de forma simultánea se ha ingerido alcohol etílico o si se aplica tempranamente el tratamiento adecuado.

\section{Tratamiento $^{10}$}

La ingestión de metanol debe considerarse siempre como una intoxicación muy grave, en la que es preferible una excesiva toma de precauciones o actuaciones a la posterior lamentación por una inhibición terapéutica. Por tanto, a la hora de tratar$\mathrm{la}^{10}$ se aconseja iniciar la administración de su antídoto (etanol) cuando sospechemos una intoxicación de metanol importante (> $30 \mathrm{ml}$ en adultos y $>0,4 \mathrm{ml} / \mathrm{kg}$ en niños) y/o ante al presencia de una acidosis metabólica y/o clínica, aunque no conozcamos la dosis ingerida ni los niveles de metanol.

\section{Lavado gástrico}

El lavado gástrico es eficaz en todos los pacientes que acudan dentro de las 1-2 horas post-ingestión. El carbón activado no es eficaz en la intoxicación por metanol, ni tampoco los laxantes o los catárticos.

\section{Etanol}

El alcohol etílico es el tratamiento antidótico de elección, aunque hoy en día se dispone de otro antídoto: fomepizol o 4metilpirazol. El etanol actúa bloqueando la formación de los dos metabolitos (formaldehído y ácido fórmico), responsables de la toxicidad, debido a su alta afinidad por la alcoholdeshidrogenasa (ADH). Para que dicho efecto se ejerza de forma adecuada, se deben mantener unos niveles de etanol en plasma de $1-1,5 \mathrm{mg} / \mathrm{ml}(100-150 \mathrm{mg} / 100$ $\mathrm{ml})$. Este tratamiento requiere la monitorización de los valores plasmáticos de etanol, ya que existen variaciones interindivi- duales en su metabolismo y hay cierta dificultad para mantener los valores adecuados, sobre todo cuando se realiza hemodiálisis junto con la administración de etanol.

El etanol puede administrarse por vía oral o intravenosa (vía central por elevada hiperosmolaridad de la perfusión), aunque se recomienda la vía intravenosa, pues estos pacientes con frecuencia padecen gastritis, náuseas y vómitos, con el consiguiente peligro de broncoaspiración. Para cualquiera de las dos vías es necesario diluir el alcohol etílico absoluto; si es por vía oral se diluye con agua hasta llegar a una concentración del $20-30 \%$, y en el caso de la vía intravenosa se diluye en suero glucosado hasta llegar al 5-10\%. Una pauta podría ser la siguiente, para una persona de $70 \mathrm{Kg}$.

- Dosis de ataque: $1 \mathrm{ml}$ de alcohol etílico absoluto por kilogramo de peso.

$1 \mathrm{ml} / \mathrm{Kg} \mathrm{x} 70 \mathrm{~kg}=70 \mathrm{ml}$ de etanol al $100 \%$.

- Vía oral: whisky 40\%: $175 \mathrm{ml}$ de whisky más 175 de agua para que la solución final tenga un concentración de etanol del $20 \%$.

- Vía intravenosa: $70 \mathrm{ml}$ de etanol al $100 \%+630$ cc de suero glucosado para que la concentración de etanol sea del $10 \%$, a pasar durante 15 minutos.

- Dosis de mantenimiento: 0,16 $\mathrm{ml} / \mathrm{Kg} /$ hora. Dicha dosis debe multiplicarse $(0,20-0,40 \mathrm{ml} / \mathrm{Kg} / \mathrm{h})$ durante el tiempo que se mantenga la hemodiálisis, si es que se aplica. Si se trata de un alcohólico crónico, la dosis de mantenimiento es de $0,2 \mathrm{ml} / \mathrm{kg} / \mathrm{h}$. La dosis de mantenimiento debe seguirse hasta que las concentraciones de metanol se encuentren por debajo de $20 \mathrm{mg} / 100 \mathrm{ml}$, y si no se dispone de este dato, hasta que el enfermo tenga un $\mathrm{pH}>7,3$ sin ayuda del bicarbonato.

- 0,16 ml/Kg/hora $\times 70 \mathrm{Kg}=11,2 \mathrm{ml} /$ hora de etanol al $100 \%$.

- Vía oral: whisky 40\%: $28 \mathrm{ml} /$ hora de whisky $+28 \mathrm{ml} /$ hora de agua. (Etanol $20 \%)$.

- Vía intravenosa: 11,2 $\mathrm{ml}$ de etanol al $100 \%$ diluidos en $100,8 \mathrm{ml}$ de glucosa- 
do, dándose $112 \mathrm{ml}$ de esa mezcla a la hora. (Etanol 10\%).

\section{Fomepizol ó 4-metilpirazol}

El fomepizol es una sustancia que inhibe competitivamente la $\mathrm{ADH}$, con una afinidad por ésta 80.000 veces superior a la del metanol y 8.000 veces superior a la del etanol, que no tiene efectos hepatotóxicos como su antecesor, el pirazole. Tiene algunas ventajas sobre el etanol: no incrementa la sedación sobre el paciente, tiene menos riesgo de hipoglucemia, menos problemas de exceso de líquidos, menos problemas en pacientes inestables hemodinámicamente, fácil manejo y administración, pudiéndose administrar tanto por vía oral como intravenosa. El gran inconveniente es su elevado precio, pues un tratamiento viene a costar entre 3.000 y $6.000 €$. Su utilización esta aprobada por la Food and Drug Administration (FDA) para las intoxicaciones agudas por metanol y etilenglicol. En España se obtiene como medicación extranjera (Antizol®): 4 viales de 1,5 $\mathrm{ml}$ con $1,5 \mathrm{~g}$ por vial; precio: $5.756,85 €$. Se administrará diluido en suero y a pasar en 30 minutos cada dosis.

- Dosis de ataque: $15 \mathrm{mg} / \mathrm{Kg}$.

- Dosis de mantenimiento: $10 \mathrm{mg} / \mathrm{Kg}$ cada 12 horas por 2 días y luego $15 \mathrm{mg} / \mathrm{Kg}$ cada 12 horas, hasta normalización del paciente.

En la actualidad y en nuestro medio asistencial, el etanol continúa siendo el tratamiento de las intoxicaciones por metanol o etilenglicol. La falta de datos definitivos sobre la eficacia y la eficiencia del fomepizol determina que no podamos introducir este efectivo antídoto en nuestros protocolos, pero sí debemos estar atentos a nuevas aportaciones que puedan surgir. La única indicación sería una contraindicación absoluta del etanol, como por ejemplo la ingestión concomitante de disulfiram, situación clínica verdaderamente excepcional.

\section{Alcalinización: la administración de bicarbonato sódico 1M}

No sólo mejora la acidosis metabólica, sino que también evita la formación de ácido fórmico. Se debe comenzar su perfusión lo antes posible, cuando el bicarbonato sea inferior a $18 \mathrm{mEq} / \mathrm{l}$. En ocasiones la cantidad necesaria de bicarbonato es elevada (500-1.000 mEq/día), ya que con relativa frecuencia los pacientes presentan un $\mathrm{pH}$ inferior a 7,0, que no responde al tratamiento con bicarbonato.

\section{Ácido fólico}

Incrementa la degradación del ácido fórmico en $\mathrm{H}_{2} \mathrm{O}$ y $\mathrm{CO}_{2}$, y puede reducir la gravedad de las lesiones oculares. Dosis: 50 $\mathrm{mg} / 6$ horas iv diluido en suero glucosado.

\section{Hemodiálisis}

Entre las medidas encaminadas a activar la extracción del metanol, la hemodiálisis es la más útil, ya que depura tanto el metanol como sus metabolitos. La diuresis forzada no es eficaz y la diálisis peritoneal apenas tiene efecto eliminador. Indicaciones de hemodiálisis:

- Concentraciones de metanol > $50 \mathrm{mg} / 100$ $\mathrm{ml}(0,5 \mathrm{~g} / \mathrm{l})$.

- Acidosis metabólica $(\mathrm{pH}<7,25)$ refractaria al tratamiento.

- Manifestaciones visuales.

- Depresión del nivel de conciencia.

- Insuficiencia renal.

La hemodiálisis debe seguirse hasta que la metanolemia sea inferior a 29 $\mathrm{mg} / 100 \mathrm{ml}$ o el pH se mantenga sin ayuda de bicarbonato por encima de 7,3. En caso de no disponer de este dato, debe continuarse durante 10-12 horas. Durante la hemodiálisis se debe seguir administrando etanol, aunque la dosis de mantenimiento sea superior. $(0,2-0,4 \mathrm{ml} / \mathrm{Kg} / \mathrm{h})$.

\section{ETILENGLICOL}

El etilenglicol es un alcohol de estructura similar al alcohol etílico, pero con la adición de un grupo hidroxilo en cada carbono. Es un líquido incoloro, inodoro y no volátil, de amplio uso industrial, tanto como intermedio de síntesis como por su tradicional uso como disolvente y típicamente como anticongelante. Otros glicoles, como el dietilenglicol, trietilenglicol y tetraetilenglicol, son también absorbibles 
por vía oral y su toxicidad es parecida. Los polietilenglicoles, con peso molecular > 200, y el propilenglicol, se absorben mal por vía digestiva, por lo que se necesitan dosis masivas o administración parenteral para inducir intoxicaciones. Por tanto, la expresión global de glicol o glicoles puede englobar sustancias muy diferentes, por lo que es necesario precisar bien el tipo exacto de producto para prever la toxicidad y decidir el tratamiento adecuado.

La causa más frecuente de intoxicación es la ingesta accidental de líquido anticongelante basado en etilenglicol. En ocasiones este producto es un adulterante de bebidas alcohólicas o es ingerido por pacientes alcohólicos como un sustituto del etanol. También algunos de estos productos se han utilizado como disolventes de productos farmacéuticos en preparaciones tópicas, lo que podría originar intoxicaciones por vía transcutánea.

\section{Fisiopatología ${ }^{7,9}$}

El etilenglicol y sus análogos, son sustancias poco tóxicas per se, hasta que son metabolizadas en el hígado, a través de la alcoholdeshidrogenasa, a glicoaldehído y, posteriormente, a ácido glicólico, ácido glioxílico y ácido oxálico, que son los responsables de la toxicidad.

El signo típico de la intoxicación es la acidosis metabólica con aumento del hiato aniónico y osmolar. A la acidosis contribuyen varios factores, incluyendo los ácidos anteriormente citados y el ácido láctico. El ácido oxálico produce depresión miocárdica y necrosis tubular aguda. El alcohol aldehído, y los ácidos glicólico y glioxílico pueden contribuir a la depresión del SNC y a la toxicidad renal, con hemorragias focales, necrosis cortical, dilatación de túbulos proximales y formación de cristales de oxalato cálcico. La quelación del calcio por el ácido oxálico, produce hipocalcemia.

\section{Clínica}

En la clínica ${ }^{7-9}$, tras la ingesta se produce una fase de euforia inicial, como en la intoxicación etílica. Posteriormente aparecen náuseas y vómitos, seguidos de depresión del SNC, asociado a edema cerebral, pudiendo aparecer diversos síntomas como confusión, alucinaciones, convulsiones, coma, rigidez de nuca, temblores, hiporreflexia y tetania. Asimismo, puede haber dolor abdominal y hematemesis. Desde el inicio de los síntomas es posible encontrar acidosis metabólica, aumento del anión gap, aumento del lactato, hipocalcemia, y en ocasiones, hipopotasemia.

Entre las 12-48 horas se afecta el aparato circulatorio y respiratorio y aparece taquicardia, taquipnea, cianosis e hipertensión arterial, edema agudo de pulmón cardiogénico y no cardiogénico, arritmias y muerte, si no se trata. Entre las 24-72 horas después de la ingestión, aparece el fallo renal, secundario a necrosis tubular, edema renal y depósito de cristales de oxalato.

\section{Diagnóstico ${ }^{7}$}

Debe sospecharse en todas las situaciones en que, de forma inexplicable, exista acidosis metabólica con aumento del hiato aniónico y osmolar, en que aparezcan los signos y síntomas comentados anteriormente. El diagnóstico se confirmará por la presencia en sangre de etilenglicol.

La evaluación de la gravedad se hace por criterio clínico, la acidosis metabólica, la hipocalcemia, y por la determinación de etilenglicol en sangre. La dosis tóxica es de 50-100 ml de ingestión del producto, y por encima de $100 \mathrm{ml}$, se considera potencialmente mortal. Una concentración de etilenglicol en sangre entre $0,2-0,5 \mathrm{~g} / \mathrm{l}$ se considera tóxica y por encima de $1 \mathrm{~g} / \mathrm{l}$, potencialmente mortal. La valoración de esta intoxicación puede hacerse también a través de las determinaciones de ácido glicólico. También es útil, la presencia de cristales de oxalato cálcico en el sedimento urinario.

\section{Tratamiento $^{11}$}

- Medidas sintomáticas y de soporte vital: corrección de la acidosis metabólica con la administración de bicarbonato sódico. Administrar calcio si las cifras de calcio están bajas o el paciente presenta manifestaciones clínicas debido a la hipocalcemia.

- Prevención de la absorción digestiva: debe practicarse lavado gástrico precoz, 
siendo eficaz sólo si se realiza dentro de las dos primeras horas post-ingesta. El carbón activado y los catárticos no son eficaces

- Medidas para frenar la metabolización del etilenglicol: el método más simple y eficaz es la administración de competidor frente a la alcoholdeshidrogenasa, que es el etanol. En todo paciente con posibilidad de haber ingerido más de 50 ml de etilenglicol, que presenta disminución del nivel de conciencia, y/o acidosis metabólica, o niveles de etilenglicol superiores a $2 \mathrm{~g} / \mathrm{l}$, está justificado el inicio del tratamiento con etanol, siguiéndose una pauta similar a la utilizada en la intoxicación por metanol. Lo mismo ocurre con el fomepizol o 4-metilpirazol.

- Medidas para aumentar la eliminación de etilenglicol: la hemodiálisis es muy eficaz. Debe iniciarse cuando existen trastornos neurológicos, acidosis metabólica severa, o niveles de etilenglicol $>0,5$ $\mathrm{g} / \mathrm{l}$. La hemodiálisis debe mantenerse hasta que el nivel plasmático de etilenglicol sea inferior a $0,1 \mathrm{~g} / \mathrm{l}$.

- Otras medidas: deben administrarse repetidas dosis de tiamina y piridoxina durante al menos 48 horas, ya que actúan como cofactores que favorecen la metabolización de los metabolitos tóxicos (ácido glioxálico).

\section{BIBLIOGRAFÍA}

1. Observatorio Español sobre Drogas (1999). Madrid: Delegación del Gobierno para el Plan Nacional sobre drogas.
2. Marruecos L, Nogué S, Nolla J. Alcoholes y glicoles. Alcohol etílico. S. Nogué. Toxicología Clínica: 171-173.

3. JONES AW. Aspects of in-vivo pharmacokinetics of etanol. Alcohol Clin Exp Res 2000; 24: 400-402.

4. Oneta CM, Simanowski UA, Martinez M, AllaliHassani A, Parés X, OMÁn N et al. First pass metabolism of ethanos is strinkgly influenced by speed of gastric emptying. Gut 1998; 43: 612-619.

5. SCHAFFER A, NARANJo CA. Recommended drug treatment strategies for the alcoholic patient. Drugs 1998; 56: 571-585.

6. ElmER GI, GEORGe FR. Antagonism of ethanol by pretreatment or posttreatment with $\mathrm{RO}$ 15-4513 and indomethacin alone or in combination. Alcohol Clin Exp Res 1995; 19: 490495.

7. MaRruecos-Sant L. Tratamiento de las intoxicaciones por metanol y etilenglicol. Med Intensiva 2002; 26: 248-250.

8. Toxicología NET (CD-ROM). Editores: Castel AN, Marruecos-Sant L, Nolla J. Universidad Autónoma de Barcelona, 2002.

9. Principios de Urgencias, Emergencias y Cuidados Críticos. Sociedad Andaluza de Medicina Intensiva y Unidades Coronarias (SAMIUC). Ed. Alhulia, 1999.

10. Barceloux DG, Bond GR, KRENZELoK EP, CoOPER $\mathrm{H}$, VALE JA. Americam Academy of Clinical Toxicology practice guidelines on the treatment of methanol poisoning. J Toxicol Clin Toxicol 2002; 40: 415-446.

11. Barceloux DG, KRenzelok EP, Olson K, Watson W. American Academy of Clinical Toxicology practice guidelines on the treatment of ethylene glycol poisoning. Ad Hoc Committee. J Toxicol Clin Toxicol 1999; 37: 537-560. 
